\title{
Isoform-Specific Substrate Inhibition Mechanism of Human Tryptophan Hydroxylase
}

Tidemand, Kasper Damgaard; Peters, Günther H.J.; Harris, Pernille; Stensgaard, Eva; Christensen, Hans Erik Mølager

\section{Published in:}

Biochemistry

Link to article, DOI:

10.1021/acs.biochem.7b00763

Publication date:

2017

Document Version

Peer reviewed version

Link back to DTU Orbit

Citation $(A P A)$ :

Tidemand, K. D., Peters, G. H. J., Harris, P., Stensgaard, E., \& Christensen, H. E. M. (2017). Isoform-Specific Substrate Inhibition Mechanism of Human Tryptophan Hydroxylase. Biochemistry, 56(46), 6155-6164. https://doi.org/10.1021/acs.biochem.7b00763

\section{General rights}

Copyright and moral rights for the publications made accessible in the public portal are retained by the authors and/or other copyright owners and it is a condition of accessing publications that users recognise and abide by the legal requirements associated with these rights.

- Users may download and print one copy of any publication from the public portal for the purpose of private study or research.

- You may not further distribute the material or use it for any profit-making activity or commercial gain

- You may freely distribute the URL identifying the publication in the public portal 


\section{Isoform-Specific Substrate Inhibition Mechanism of Human Tryptophan Hydroxylase}

Kasper D. Tidemand, Günther H. Peters*, Pernille Harris, Eva Stensgaard, and Hans E. M.

Christensen*.

Department of Chemistry, Technical University of Denmark, Kemitorvet 207, DK-2800 Kgs. Lyngby, Denmark. 


\begin{abstract}
Tryptophan hydroxylase (TPH) catalyzes the initial and rate-limiting step in the biosynthesis of serotonin, which is associated with a variety of disorders such as depression and irritable bowel syndrome. TPH exists in two isoforms; TPH1 and TPH2. TPH1 catalyzes the initial step in the synthesis of serotonin in the peripheral tissues, while TPH2 catalyzes this step in the brain. In this study, the steady-state kinetic mechanism for the catalytic domain of human TPH1 has been determined. Varying substrate tryptophan (Trp) and tetrahydrobiopterin $\left(\mathrm{BH}_{4}\right)$ results in a hybrid Ping Pong-ordered mechanism in which the reaction can either occur through a Ping Pong or a sequential mechanism depending on the concentration of tryptophan. The catalytic domain of TPH1 shares a sequence identity of $81 \%$ with TPH2. Despite the high sequence identity, differences in the kinetic parameters of the isoforms have been identified, i.e. only TPH1 displays substrate tryptophan inhibition. This study demonstrates that the difference can be traced to an active site loop which displays different properties in the TPH isoforms. Steady-state kinetic results of the isoforms, and variants with point mutations in a loop lining the active site, show that the kinetic parameters of only TPH1 are significantly changed upon mutations. Mutations in the active site loop of TPH1 result in an increase in the substrate inhibition constant, $K_{i}$, and therefore turnover rate. Molecular dynamics simulations reveal that this substrate inhibition mechanism occurs through a closure of the co-substrate, $\mathrm{BH}_{4}$, binding pocket, which is induced by Trp binding.
\end{abstract}




\section{Introduction}

Tryptophan hydroxylase (TPH) catalyzes the first and rate-limiting reaction in the biosynthesis of the hormone and neurotransmitter serotonin $(5-\mathrm{HT})$. TPH uses $\mathrm{Fe}^{2+}$, tetrahydrobiopterin $\left(\mathrm{BH}_{4}\right)$, and $\mathrm{O}_{2}$ to generate 5-hydroxytryptophan (5-HTP) by hydroxylation of tryptophan (Trp). ${ }^{1}$ 5-HTP is converted to 5-HT by aromatic amino acid decarboxylase ${ }^{2,3}$. Together with phenylalanine hydroxylase (PAH) and tyrosine hydroxylase (TH), TPH is a member of the enzyme subfamily of iron(II)-containing monooxygenases which are known as aromatic amino acid hydroxylases (AAAHs). The enzymes in the AAAH family form tetramers, in which each monomeric subunit consists of an N-terminal regulatory domain, a highly conserved catalytic domain, and a C-terminal tetramerization domain. ${ }^{1,4}$ In human two isoforms of TPH exist, where isoform 1 (TPH1) mainly is responsible for catalyzing the initial step in the biosynthesis of peripheral serotonin, and isoform 2 (TPH2) is associated with biosynthesis of neuronal serotonin ${ }^{1}$. Dysregulation of serotonin levels is involved in various physiological and psychiatric disorders such as irritable bowel syndrome, depression, obsessive compulsive disorder, and schizophrenia ${ }^{5}$. Decreased levels of neuronal serotonin are associated with neurological disorders, whereas some gastrointestinal disorders are associated with increased peripheral serotonin levels ${ }^{6-8}$.

The kinetic mechanism has not unambiguously been determined for the aromatic amino acid hydroxylases. Despite great efforts to dissect the mechanism, ambiguities still exist. Of the AAAHs, PAH and TH have been more thoroughly studied. Steady-state kinetic studies have shown that bovine TH follows a Ping Pong mechanism. ${ }^{9,10}$ This mechanism is supported by observation that in rat $\mathrm{TH}$, where oxidation of $\mathrm{BH}_{4}$ can occur in the absence of substrate tyrosine at $\mathrm{pH} 8.2 .{ }^{11}$ However, the oxidation occurs at slower rates at $\mathrm{pH}$ 7.2 and not at all at $\mathrm{pH}$ 6.5. ${ }^{11,12}$ In contrast, another steady-state kinetic study on rat TH finds an ordered sequential mechanism involving a quaternary complex with all three substrates bound. ${ }^{12}$ The presence of a ternary complex which binds $\mathrm{O}_{2}$ during catalysis in rat $\mathrm{TH}$, is supported by a spectroscopic study. ${ }^{13}$ Furthermore, a sequential mechanism is supported by single turn-over kinetic studies on PAH from chromabacterium violaceum ${ }^{14,15}$ and rat $^{16}$. Similar results have been obtained for rabbit TPH1. ${ }^{17}$ The substrate binding order in the AAAHs have been established to occur in an ordered fashion where pterin binds followed by substrate amino acid and then $\mathrm{O}_{2} .{ }^{9,15,16}$ That pterin binds as the first substrate is consistent with finding in TPH1 where inhibitors display uncompetitive inhibition towards 6- $\mathrm{MePH}_{4}$, which indicates that pterin binds first. ${ }^{18}$ Additionally, the crystal structure of chicken TPH (pdb: 3E2T) shows that binding of tryptophan and possibly imidazole results in a closed conformation, which indicates the formation of a deadend complex. ${ }^{19}$ Despite a sequence identity of $71 \%$, the two isoforms of TPH display differences in their kinetic properties. ${ }^{20,21}$ Some of the differences are accounted for by the N-terminal regulatory domains. TPH2 has an additional 46 residues in the regulatory domain compared to TPH1. This terminal extension has a great impact on enzymatic activity, and serine 19 in the terminus of TPH2 has been identified as a phosphorylation site. ${ }^{22}$ However, even in the absence of the regulatory domains, the isoforms display 
different kinetic properties. ${ }^{21}$ Besides differences in kinetic parameters ( $K_{\mathrm{m}}$ and $V_{\max }$-values), TPH1 displays a greater degree of substrate tryptophan inhibition compared to TPH2. ${ }^{20,}{ }^{21}$ Human TPH1 variants without the regulatory or tetramerization domain exhibit substrate inhibition comparable to full-length TPH1. ${ }^{23}$ The underlying molecular mechanism of this inhibition and the differences in the isoforms that govern these observations remains elusive.

Here, we describe results that identify the steady-state kinetic mechanism for the catalytic domain of human TPH1. Furthermore, we describe results that identify the structural origin of the competitive substrate inhibition mechanism. The inhibition mechanism of TPH1 is governed by the orientation of Tyr125 which enables the active site loop to participate in the mechanism. Participation of Tyr125 occurs through the adjacent Tyr235 which is found to block the active site access upon substrate Trp binding.

\section{Experimental Procedures}

Materials. All used chemicals were of analytical grade, and all solutions were prepared using water from an 18.2 M $\Omega \cdot \mathrm{cm}$ Milli-Q synthesis A10 Q-Gard system which was filtered through a $0.22 \mu \mathrm{m}$ filter. Protein purification was performed on an ÄKTA purifier 100 from GE Healthcare. Utilized GE Healthcare column are: HiLoad Superdex 200 26/60 pg, Superdex 200 10/300 GL, and a XK 16/20 column packed with 25 mL Dextrin Sepharose High Performance media. During purifications, all TPH containing solutions were kept in ice water, except during the chromatographic steps, which were performed at room temperature. Protein solutions were concentrated using an Amicon ultrafiltration cell with an Ultracell PL-3 membrane. Protein concentrations were determined by measuring the absorbance at $280 \mathrm{~nm}$ on an ND-1000 NanoDrop Spectrophotometer from Saveen Werner.

Cloning and Expression. Full-length human TPH2 cDNA optimized for expression in E. coli was obtained from GenScript. The construct encoding the different protein variants was obtained by PCR. All DNA sequences were verified by sequencing (Eurofins). All proteins were expressed as maltose binding protein fusion proteins from the pET26 expression vector in E. coli BL21(DE3) (Novagen) cells. All TPH1 and TPH2 variants were expressed as $\mathrm{N} \Delta 99 / \mathrm{C} \Delta 31$ and $\mathrm{N} \Delta 145 / \mathrm{C} \Delta 31$, respectively, lacking the $\mathrm{N}$-terminal regulatory domain and the C-terminal tetramerization domain. The sequences of the proteins expressed are given in Supporting Information. The recombinant fusion proteins contain a cleavage recognition site for human rhinovirus $3 \mathrm{C}$ protease $(3 \mathrm{CP})^{24}$. Proteins were expressed at $20^{\circ} \mathrm{C}$ for $14 \mathrm{~h}$ as previously described ${ }^{25}$. MBP-3CP was cloned, expressed, and purified in-house.

Purification. The purification procedure comprises an affinity chromatography step utilizing a column packed with dextrin sepharose and a size exclusion step using a HiLoad Superdex 200 prep grade column, as previously described ${ }^{26}$. All variants were cleaved from the MBP fusion protein utilizing an on-column cleavage procedure with MBP-3CP during the first purification step. Human TPH1 variants were purified in 
buffer containing $20 \mathrm{mM}$ TRIS/ $\mathrm{H}_{2} \mathrm{SO}_{4}, 100 \mathrm{mM}\left(\mathrm{NH}_{4}\right)_{2} \mathrm{SO}_{4}$, $\mathrm{pH} 8.0$ and human TPH2 variants in $20 \mathrm{mM}$ HEPES/NH $4 \mathrm{OH}, 100 \mathrm{mM}\left(\mathrm{NH}_{4}\right)_{2} \mathrm{SO}_{4}$, $\mathrm{pH}$ 7.0.

Activity Assay. The activity assay was performed as described by Moran et al. ${ }^{27}$ and Nielsen et al. ${ }^{25}$, and the sample preparation was conducted as previously described ${ }^{26}$. The activity measurements were performed using a Varian Cary Eclipse Fluorescence Spectrophotometer. For activity measurements, the TPH variants were thawed under running water, filtered, and the concentrations were determined by UV-Vis absorption at $280 \mathrm{~nm}$. The TPH samples were diluted with the buffer in which they were purified. TPH activity was assayed in a reaction mixture (10x10 mm QS quartz cuvette from Hellma - $2500 \mu \mathrm{L}$ total volume) containing $50 \mathrm{mM}$ HEPES/ $\mathrm{NH}_{4} \mathrm{OH}, 200 \mathrm{mM}\left(\mathrm{NH}_{4}\right)_{2} \mathrm{SO}_{4}, \mathrm{pH} 7.0,0.025 \mathrm{~g} / \mathrm{L}$ catalase, $25 \mu \mathrm{M}\left(\mathrm{NH}_{4}\right)_{2} \mathrm{Fe}(\mathrm{II})\left(\mathrm{SO}_{4}\right)_{2} \cdot 6 \mathrm{H}_{2} \mathrm{O}, 7$ $\mathrm{mM}$ dithiothreitol (DTT) with stirring at $15{ }^{\circ} \mathrm{C}$. The concentration of TPH was $0.8 \mu \mathrm{M}$ in all measurements except for Trp at 1.5 and $2.5 \mu \mathrm{M}$, where the concentrations were 0.24 and $0.4 \mu \mathrm{M}$, respectively. The standard substrate concentrations were $70 \mu \mathrm{M} \mathrm{L}-\mathrm{Trp}, 300 \mu \mathrm{M} \mathrm{BH}$, and $500 \mu \mathrm{M} \mathrm{O}$. The $\mathrm{O}_{2}$ concentration was achieved by equilibrating the solution with a mixture of $\mathrm{O}_{2}$ and $\mathrm{N}_{2}$ as previously described. ${ }^{25}$ Excitation and emission wavelengths were 300 and $330 \mathrm{~nm}$, respectively. Initial velocity was determined from linear regression through the first 0.04 min after $\mathrm{BH}_{4}$ addition. Apparent kinetic parameters were calculated by curve fitting using non-linear least-squares approach. When only one substrate was varied, the initial velocity data were fitted using eq. 1 or eq. 2 in case of substrate inhibition. When two substrates were varied, the initial velocity data were fitted using eq. $3 .{ }^{28,29,30}$

$v_{i}=\frac{V_{\max }[S]}{K_{m}+[S]}$

$v_{i}=\frac{V_{\max }[S]}{K_{m}+[S]+\frac{[S]^{2}}{K_{i}}}$

$v_{i}=\frac{V_{\max }[A][B]+V_{2}[A][B]^{2}}{K_{m, A}[B]\left(1+\frac{[B]}{K_{i}}\right)+K_{m, B}[A]+[A][B]+K_{3}[B]^{2}+K_{4}[A][B]^{2}}$

Kinetic parameters were determined from three independent experiments from at least two different purifications batches for each variant.

Molecular Dynamic Simulations. The x-ray structures of TPH1 (pdb: 1MLW, open loop conformation) and TPH2 (pdb: 4V06, open loop conformation) were used as initial models for MD simulations. The crystal structure of human TPH1 lacks interpretable electron density in the C-terminus (residue 394 to 413). Homology modeling was utilized to build the terminus with 4V06 as template. The position and orientation of substrate Trp were obtained from the crystal structure of chicken TPH (pdb: 3E2 $\mathrm{T}^{19}$ ) and superimposed into the structures of human TPH isoforms. In the Schrödinger suite, mutations were performed by Residue 
Scanning in the BioLuminate suite. ${ }^{31}$ The proteins were prepared for simulation with the default parameters of the Protein Preparation Workflow in Maestro ${ }^{32}$. Protonation states at $\mathrm{pH} 8.0$ were assigned with the PropKa module ${ }^{33}$. The system was built by adding $0.3 \mathrm{M} \mathrm{NaCl}$ and water molecules (TIP3P model ${ }^{34}$ ) in an orthorhombic box exceeding $10 \AA$ in each direction from the protein using System Builder workflow in Desmond $^{35}$. The MD simulations were performed with the Desmond package using the OPLS3 force-field ${ }^{36}$, and periodic boundary conditions in all three Cartesian coordinates were applied. Particle-mesh Ewald method ${ }^{37}$ was applied to calculate long-range electrostatic interactions. Short-range electrostatic and van der Waals interactions were smoothly truncated at $9 \AA$. The simulations were run with constant number of atoms, with constant temperature of $300 \mathrm{~K}$ maintained with Nosé-Hoover thermostats ${ }^{38}$, and constant pressure maintained at 1.01325 bar with the Martyna-Tobias-Klein barostat method ${ }^{39}$. RESPA integrator ${ }^{40}$, with a 2.0-fs time step was utilized to solve the equations of motion for bonded and short-range interactions. The RESPA integrator default setting for nonbonded interactions beyond the 0.9-nm cutoff was 6.0-fs time step. Before every 100-ns production simulation run, a relaxation with the default parameters in Desmond was performed. Configurations and energies were saved at 5-ps intervals. Convergences of the MD simulations were based on RMSD calculations.

\section{Results}

Enzymatic -Mechanism - To explore the kinetic mechanism of TPH1, the initial velocities of the catalytic domain of human TPH1 (chTPH1) was determined at various tryptophan and $\mathrm{BH}_{4}$ concentrations. The concentrations of the substrates were varied in a concentration matrix of 14 tryptophan concentrations and six $\mathrm{BH}_{4}$ concentrations. The $\mathrm{O}_{2}$ concentration was $500 \mu \mathrm{M}$ in all measurements and apparent kinetic parameters were hence derived. This data is presented in Figure 1. For clarity, only the lowest seven concentrations of tryptophan are shown in Figure 1B. (For all concentrations see Supporting Information Figure S1). Figure 1A shows that the data deviate from the classical hyperbolic curve. The data can be fitted very well with the Michaelis-Menten equation with a substrate inhibition term (eq. 2) (see Figure S2), which describes linear substrate inhibition resulting from a dead-end complex formation between substrate and a non-productive enzyme form. ${ }^{41}$ When the data in Figure $1 \mathrm{~A}$ are replotted as $v_{0}$ versus $\left[\mathrm{BH}_{4}\right]$, the plots in Figure $1 \mathrm{~B}$ are obtained. The data follow a classic hyperbolic shape, and the Michaelis-Menten equation (eq. 1) fits well to the data (Figure S3). By fitting eq. 2 to the data for each fixed $\mathrm{BH}_{4}$ concentration, apparent tryptophan substrate inhibition constants, $K_{i}$, can be determined. The secondary plot in Figure 2 shows that chTPH1 displays $\mathrm{BH}_{4}$ concentration-dependent substrate inhibition. For instance, at low fixed concentrations of $\mathrm{BH}_{4}(20 \mu \mathrm{M})$, chTPH1 has an apparent $K_{i}$ of $24 \mu \mathrm{M}$, while at high $\mathrm{BH}_{4}$ concentration $(400 \mu \mathrm{M})$ the apparent $K_{i}$ is increased to $206 \mu \mathrm{M}$. To evaluate which mechanism the kinetic data represents, global fitting was conducted. The datasets were fitted to the velocity equations describing a $i$ ) Ping Pong mechanism with a dead-end EB complex formation, ii) sequential mechanism with a dead-end EB complex formation, iii) sequential mechanism with dead-end $\mathrm{EA}_{2}$ complex formation, and iv) hybrid Ping Pong-ordered mechanism 
with dead-end EB complex formation (hybrid EB). ${ }^{42}$ The hybrid Ping Pong-ordered mechanism in Figure 3 was included in the analysis because both Ping Pong and sequential mechanisms have been observed in steady-state kinetic studies of the AAAHs. ${ }^{9,10,12}$ In the hybrid model ${ }^{28,29}$, the reaction can either occur through a Ping Pong or a sequential mechanism depending on the concentration of tryptophan. Substrate inhibition is caused by competitive formation of a dead-end EB (TPH•Trp) complex formed when tryptophan binds before $\mathrm{BH}_{4}$. Such hybrid mechanisms have also been observed in e.g. pyruvate carboxylase, glutathione reductase, hypoxanthine phosphoribosyltransferase, and glutathione S-transferase A. ${ }^{30,43-45}$ In the global fit, the sequential mechanisms (ii and iii) resulted in identical fits, as $K_{\mathrm{A}}$ was reduced to zero resulting in a reduction of the equations to that of a Ping Pong mechanism (i). For this reason, identical kinetic parameters were obtained for the three mechanisms, and the sequential mechanisms were discarded. Based on this analysis of the conventional non-branched mechanisms, a Ping Pong mechanism fit the total dataset best. The hybrid EB model, however, results in an improved global fit $(p<0.01)$ compared to that of a Ping Pong mechanism, Table 1. Fitting this model to the data results in a redundancy of $K_{4}$. In the proposed hybrid mechanism in Figure 3, the order of pterin and substrate binding is in agreement with the majority of studies conducted on the AAAHs. ${ }^{9-11,15-17}$ Substrate inhibition occurs through competitive inhibition by tryptophan versus $\mathrm{BH}_{4}$. This yields a $\mathrm{BH}_{4}$ concentration-dependent substrate tryptophan inhibition constant which has similarly been observed for $\mathrm{TH}^{10}$. A hybrid Ping Pong-ordered model in which tryptophan binds before $\mathrm{BH}_{4}$ also resulted in an improved global fit $(\mathrm{p}<0.01)$ compared to that of a Ping Pong EB mechanism. This model was, however, discarded because of the accumulated experimental data, that evidently find that $\mathrm{BH}_{4}$ binds as the first substrate. ${ }^{9-11,15-17}$ Biphasic data was obtained by Oka et al. ${ }^{10}$ who therefore analyzed the data separately for velocity data obtained at respectively higher or lower than $0.2 \mathrm{mM} \mathrm{BH}_{4}$. Such data might be explained by a hybrid Ping Pong-ordered model, which follows different mechanisms depending on substrate concentration. According to this proposed hybrid model, the reaction will occur through a sequential mechanism at high tryptophan concentrations. This correlates well with the fact that several of the AAAH studies which obtain a sequential mechanism were conducted with relatively high substrate concentrations (typically $>0.5 \mathrm{mM}$ range). ${ }^{13,14,16,17}$ 


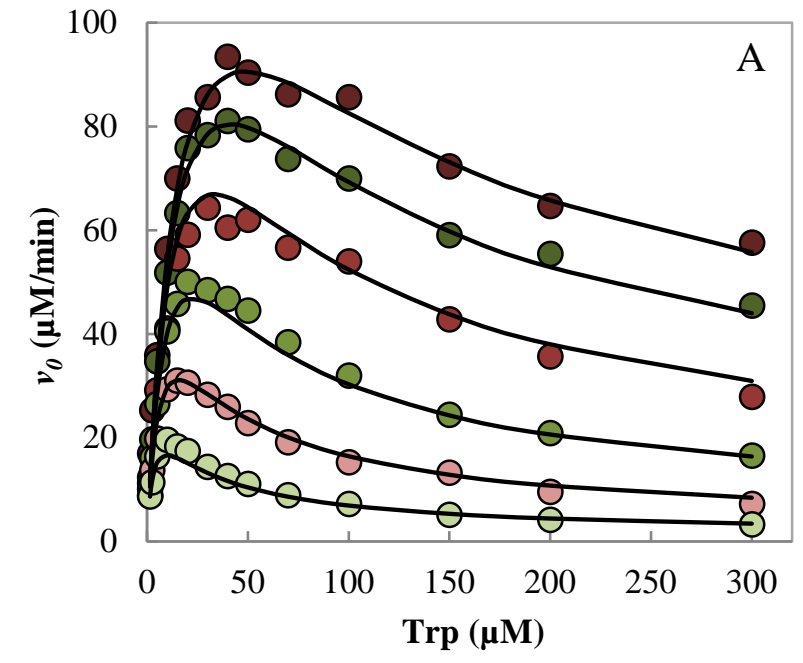

○ $400 \mu \mathrm{MBH}_{4} \bigcirc 300 \mu \mathrm{MBH}_{4}$ О $200 \mu \mathrm{MBH}_{4}$ О $100 \mu \mathrm{MBH}_{4}$ О $50 \mu \mathrm{MBH}_{4} \quad \mathrm{O} 20 \mu \mathrm{M} \mathrm{BH}_{4}$

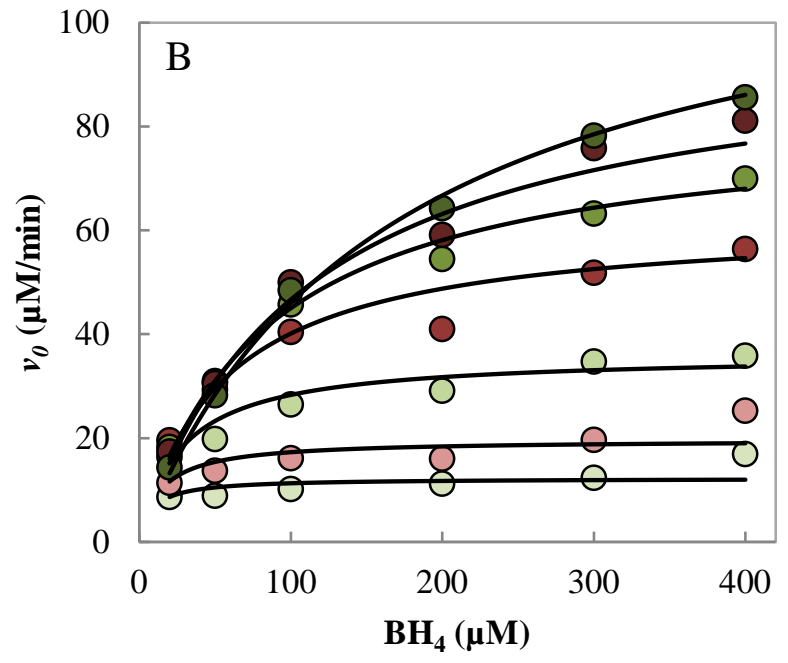

$01.5 \mu \mathrm{M}$ Trp $02.5 \mu \mathrm{M}$ Trp

О5 $\mu \mathrm{M}$ Trp $010 \mu \mathrm{M}$ Trp

○ $15 \mu \mathrm{M}$ Trp $\bigcirc 20 \mu \mathrm{M}$ Trp

Figure 1. Global fits to initial velocity measurements for chTPH1. The oxygen concentration was $500 \mu M$ in all measurements. (A) Initial velocities versus Trp concentration at fixed concentration of $\mathrm{BH}_{4}$. (B) Initial velocities versus $\mathrm{BH}_{4}$ concentration at fixed concentration of Trp. The plots are fitted with eq. 3 (Hybrid Ping Pong-ordered EB mechanism).

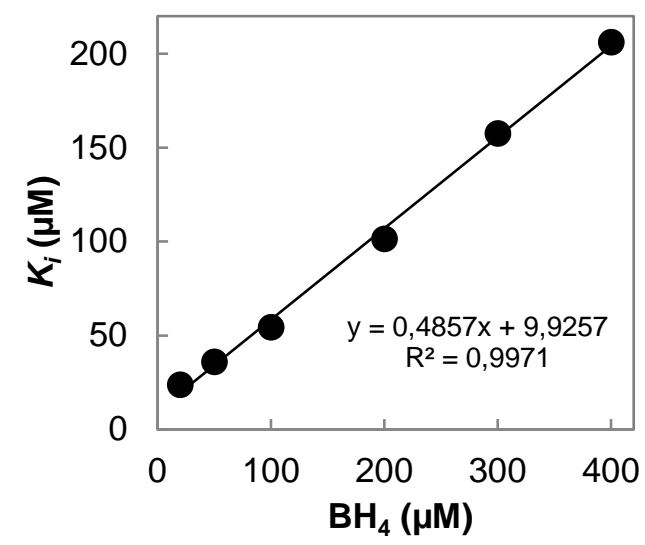

Figure 2. Secondary plot of apparent $K_{i}$-values obtained at different $\mathrm{BH}_{4}$ concentrations. Values obtained by fitting the $v 0$ vs. Trp concentration data at fixed $\mathrm{BH}_{4}$ concentrations presented in Figure 1A with eq. 2

Table 1. Kinetic parameters derived from global fits. Parameters are derived by fitting the data with a Ping Pong mechanism with dead-end EB and a hybrid Ping Pong-ordered mechanism with dead-end EB formation (Hybrid EB - eq. 3) to all the data. The model error is given by the sum of squared residuals. *: F-test, $p<0.01$.

\begin{tabular}{lllllllll}
\hline Mechanism & $\begin{array}{l}\boldsymbol{V}_{\mathbf{m a x}} \\
\boldsymbol{\mu M} \mathbf{m i n}^{-\mathbf{1}}\end{array}$ & $\begin{array}{l}\boldsymbol{V}_{\mathbf{2}} \\
\mathbf{m i n}^{\mathbf{1}}\end{array}$ & $\begin{array}{l}\boldsymbol{K}_{\mathbf{m}, \mathbf{T r}} \\
\boldsymbol{\mu M}\end{array}$ & $\begin{array}{l}\boldsymbol{K}_{\mathbf{m}, \mathbf{B H} \mathbf{4}} \\
\boldsymbol{\mu M}\end{array}$ & $\begin{array}{l}\boldsymbol{K}_{\mathbf{i}} \\
\boldsymbol{\mu M}\end{array}$ & $\boldsymbol{K}_{\mathbf{3}}$ & $\begin{array}{l}\boldsymbol{K}_{\mathbf{4}} \\
\mathbf{\mu M}^{\mathbf{1}}\end{array}$ & $\mathbf{E r r o r}$ \\
\hline Ping Pong EB & 213 & - & 23 & 181 & 51 & - & - & 551 \\
Hybrid EB & 210 & 0.3 & 24 & 135 & 52 & 2.8 & 0 & $445^{*}$ \\
\hline
\end{tabular}




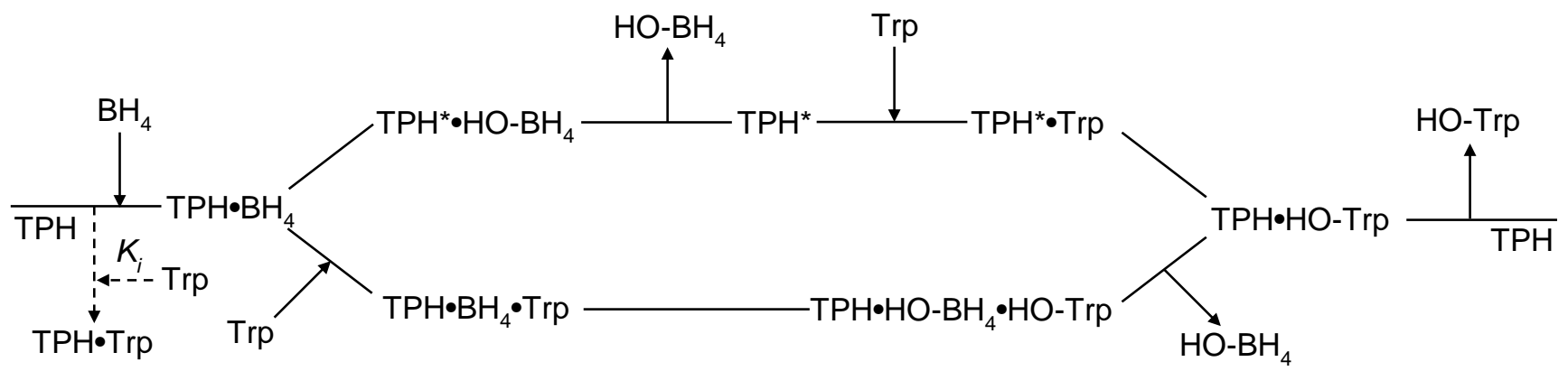

Figure 3. Basic scheme for a branching mechanism of chTPH1. The scheme corresponds to a hybrid Ping Pong-ordered mechanism with dead-end TPH•Trp complex (EB). TPH* indicates a TPH that has undergone an irreversible change (e.g. formation of $\mathrm{Fe}^{\mathrm{IV}}=\mathrm{O}$ ). Upper path represents a Ping Pong mechanism, and the lower path represents a sequential mechanism.

Sequence and Structural Analysis of Isoform Differences - The human TPH isoforms display significantly different kinetic parameters, and only TPH1 is subjected to substrate inhibition. ${ }^{21}$ In this study, it was investigated how inhibition only apply to chTPH1. The isoforms of TPH have a sequence identity of $81 \%$ in the catalytic domain, which is reflected in their similar tertiary structures. The crystal structures of the catalytic domains of human TPH1 (chTPH1 - pdb: 1MLW ${ }^{46}$ ) and human TPH2 (chTPH2 - pdb: 4V06) display a root mean square deviation of the $\mathrm{C}_{\alpha}$-atoms $\left(\mathrm{RMSD}_{\mathrm{C} \alpha}\right)$ of $1.1 \AA$. The TPH structures have $\mathrm{BH}_{2}$ and imidazole bound in the pterin binding pocket, respectively. Crystal structures of the homologous phenylalanine hydroxylase have shown that binding of $\mathrm{BH}_{2}$ or $\mathrm{BH}_{4}$ do not cause structural changes. ${ }^{47,48}$ The two TPH structures are therefore comparable. It was found that the $19 \%$ non-conserved residues are randomly distributed mainly on the surface of the protein. Within $10 \AA$ of the active site iron, only 2 out of 37 residues are not conserved, and these residues are distant from the binding pockets of the substrate and co-substrate (Supporting Information, Figure S4). As the non-conserved residues are not clustered in one area and are not close to the substrate or co-substrate binding sites, differences in the crystal structures determined by $\mathrm{RMSD}_{\mathrm{C} a}$ were analyzed. The alignment, with color-coded RMSD $\mathrm{C} \alpha$-values, in Figure 4 shows that a stretch of sequence, corresponding to a loop over the active site, displays relatively high $\mathrm{RMSD}_{\mathrm{C} \alpha^{-}}$ values compared to the rest of the structure despite a high sequence homology. The loops span residues 117137 of TPH1 and 163-183 of TPH2 and are shown in Figure 5. Especially tyrosine 125/171 in the center of the loop displays a relatively high $\mathrm{RMSD}_{\mathrm{C} \alpha}$-value (5.2 $\AA$ ). The corresponding loop in PAH has been found to reside in either an open or closed conformation depending on the active site occupancy. A closed conformation has only been observed in the presence of both a pterin and a substrate analogue. ${ }^{49,50}$ However, a structure of PAH with only a substrate/substrate analogue has not been determined, and it is therefore unknown whether the loop closure occurs solely upon binding of substrate or a combination of substrate and co-substrate. Compared to the closed structures of PAH, both isoforms of human TPH exist in open conformations. For TPH, a closed conformation has been observed in a crystal structure of chicken 
TPH1 with bound substrate Trp and imidazole (pdb: 3E2T). ${ }^{19}$ From Figure 5, it is noticed that the side chain of the tyrosine residue is oriented towards the active site in TPH1 but is pointing away from the active site in TPH2. The structures of the loops and the orientation of the tyrosine residues in the crystal structures are assessed by electron density in the loops. The $2 \mathrm{~F}_{\mathrm{o}}-\mathrm{F}_{\mathrm{c}}$ electron density maps of the crystal structures show that the structures of the loops in both isoforms are well resolved (Supporting Information Figure S5 and S6).

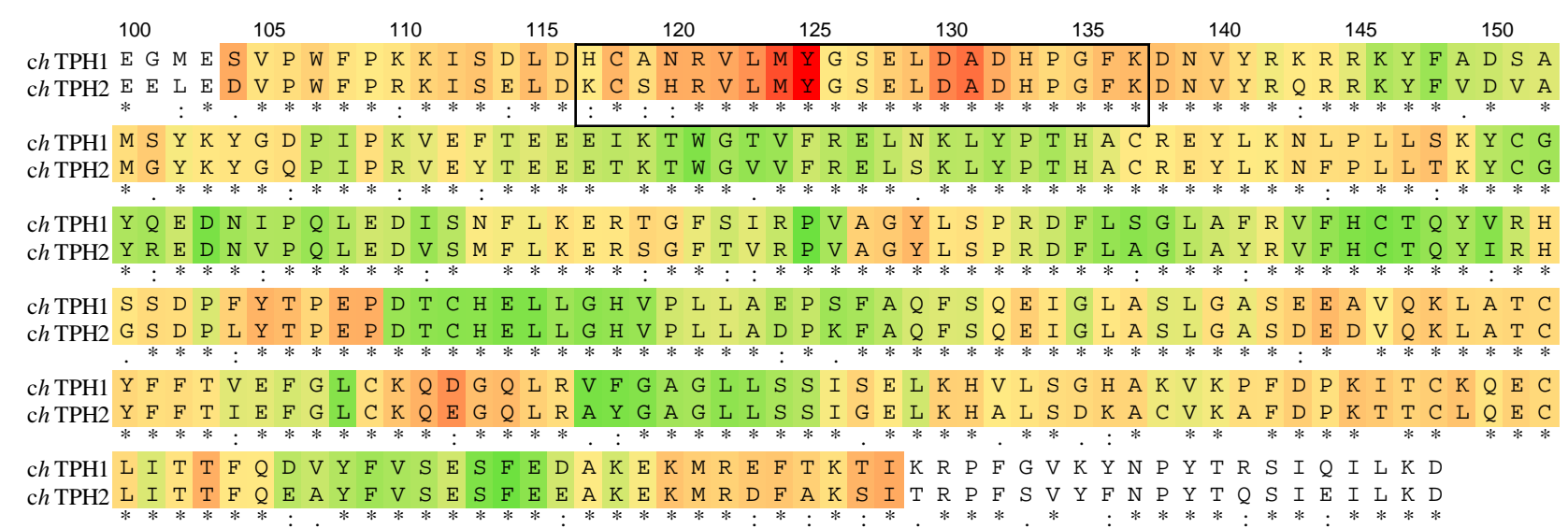

0

Figure 4. Sequence alignment of the catalytic domains of TPH1 and TPH2 (Clustal Omega 1.2.3 - * = identical, : = very similar, . = similar). The color scale (green is low and red is high) in the alignment shows the RMSD of the Ca-atoms calculated between chTPH1 (pdb: 1MLW) and chTPH2 (pdb: 4V06). The black box indicates the sequence of the active site loop. The numbering of the sequence is based on TPH1.

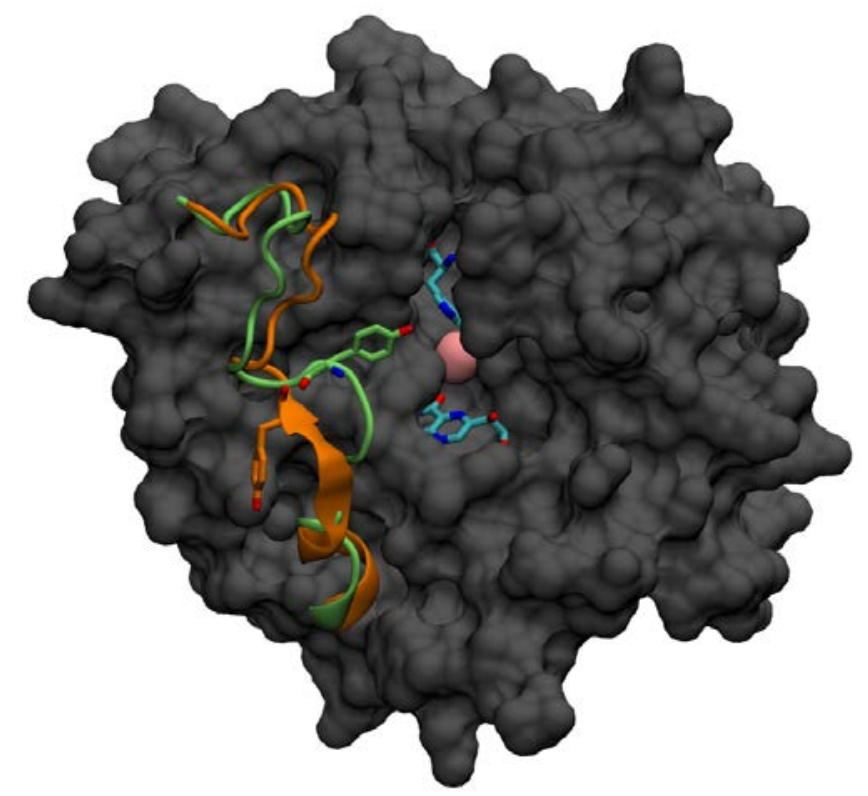

Figure 5. Crystal structure of chTPH1 (pdb: 1MLW - open conformation) with gray surface. The active site loop of chTPH1 is shown in green with tyrosine 125 displayed as sticks and the loop of chTPH2 (superimposed from pdb: 4V06 - open conformation) is shown in orange with tyrosine 171 displayed as sticks. For illustration, Trp (superimposed from pdb: 3E2T) and $\mathrm{BH}_{2}$ have been placed in their respective binding pockets. Trp and $\mathrm{BH}_{2}$ are shown as cyan sticks and iron is shown as sphere. 
The Effect of Tyr125/171 Point Mutations on TPH Kinetics - In order to probe the importance of the loop in TPH, Tyr125 in chTPH1 and Tyr171 in chTPH2 were mutated to the smaller amino acid alanine or the more bulky amino acid tryptophan and characterized for changes. As the oligomeric state and the transition temperatures of the isoform mutant variants were not changed (Supporting Information, Figure S7, Table S1, and Table S2), the kinetic parameters can be directly compared. Apparent kinetic parameters are determined given that saturated concentrations of the non-varied substrate could not be obtained due to substrate inhibition. In agreement with Windahl et al. ${ }^{21}$, chTPH1 displays substrate inhibition, with a substrate inhibition constant of $165 \pm 18 \mu \mathrm{M}$, while chTPH2 does not display substrate inhibition (Table 2, Figure 6 panel A and C open circles). Additionally, chTPH1 displays a 10-fold higher $K_{\mathrm{m}, \mathrm{BH} 4}$ compared to chTPH2. The kinetic analysis shows that mutation of the tyrosine residue in the loop results in significant changes in the kinetic parameters of chTPH1 (Table 2 and Figure 6). Mutation to alanine results in a small reduction of $K_{\mathrm{m}, \mathrm{BH} 4}$, while mutation to tryptophan results in a 5-fold reduction. A change in $K_{\mathrm{m} \text {,Trp }}$ is only observed for Y125W-chTPH1. Notably, the mutations result in a significant increase in the substrate inhibition constant, $K_{i}$. Mutation to alanine increases the $K_{\mathrm{i}}$-value slightly, while mutation to tryptophan results in a greater than 3-fold increase. The decreased substrate inhibition is clearly visualized in Figure 6 panel A. Beside the reduced $K_{\mathrm{m}, \mathrm{BH} 4}$ and increased $K_{\mathrm{i}}$-values, $k_{\text {cat,Trp }}$ is also found to change with the mutations. Similar to the other kinetic parameters, $k_{\text {cat,Trp }}$ is slightly increased with mutation to alanine and increased 3-fold by mutation to tryptophan. For chTPH2, mutation from tyrosine to alanine or tryptophan results in no significant changes in the kinetic parameters, apart from a change in the $K_{\mathrm{m}, \mathrm{Trp}}$. These observations correlate well with the different orientations of the tyrosine residue seen in the two crystal structures. In chTPH1, the tyrosine residue is in vicinity of the active site and influences the kinetics of the enzyme, while in chTPH2, the tyrosine residue point away from the active site which results in a less pronounced effect on the kinetics.

Table 2. Kinetic parameters of chTPH1 and chTPH2 variants derived from data presented in Figure 6. Parameters are given with standard deviations of at least three independent experiments from at least two purification batches. *: $p<0.05$, **: $p<$ 0.01 .

\begin{tabular}{|c|c|c|c|c|c|}
\hline \multirow[b]{2}{*}{ Variant } & \multicolumn{3}{|c|}{ Tryptophan } & \multicolumn{2}{|c|}{$\mathrm{BH}_{4}$} \\
\hline & $k_{\text {cat }}\left(\min ^{-1}\right)$ & $K_{\mathrm{i}}(\mu \mathrm{M})$ & $K_{\mathrm{m}}(\mu \mathrm{M})$ & $K_{\mathrm{m}}(\mu \mathrm{M})$ & $k_{\text {cat }}\left(\min ^{-1}\right)$ \\
\hline chТРН1 & $108 \pm 10$ & $165 \pm 18$ & $12.7 \pm 1.4$ & $285 \pm 15$ & $145 \pm 17$ \\
\hline Y125A-chTPH1 & $164 \pm 10^{* *}$ & $227 \pm 21 *$ & $12.6 \pm 0.5$ & $188 \pm 19 * *$ & $192 \pm 13 *$ \\
\hline Y125W-chTPH1 & $315 \pm 55^{* *}$ & $544 \pm 126 * *$ & $36.7 \pm 4.6^{* *}$ & $57 \pm 5^{* *}$ & $203 \pm 9 *$ \\
\hline Loop-swap-chTPH1 & $178 \pm 12^{* *}$ & $189 \pm 27$ & $13.9 \pm 2.7$ & $240 \pm 42$ & $191 \pm 18 *$ \\
\hline chТРН2 & $258 \pm 20$ & - & $13.1 \pm 1.3$ & $26.7 \pm 0.9$ & $239 \pm 12$ \\
\hline Y171A-chTPH2 & $220 \pm 9$ & - & $12.1 \pm 1.3$ & $31.1 \pm 6.0$ & $201 \pm 27$ \\
\hline Y171W-chTPH2 & $315 \pm 34$ & - & $21.7 \pm 1.5^{* *}$ & $19.2 \pm 3.4$ & $274 \pm 16$ \\
\hline Loop-swap-chTPH2 & $212 \pm 4$ & $497 \pm 54$ & $14.0 \pm 0.7$ & $37.6 \pm 8.1$ & $145 \pm 8^{* *}$ \\
\hline
\end{tabular}


Loop-Swap Mutations of Non-Conserved Residues - Of the 21 residues located in the loop, only three residues are not conserved between the isoforms (Figure 4). To examine whether these residues contribute to the different orientations of the tyrosine residue, the three non-conserved residues in chTPH1 were mutated to the corresponding residues of the sequence of chTPH2 and vice versa. In chTPH1, His117, Ala119, and Asn120 were mutated to Lys, Ser, and His, respectively (referred to as loop-swap-chTPH1), while the opposite mutations were performed in position 163, 165, and 166 of chTPH2 (referred to as loop-swapchTPH2). The kinetic results of the loop-swap mutations are presented in Figure 6, and the kinetic parameters are summarized in Table 2. In chTPH1, the loop-swap mutations result in only modest changes in the kinetic parameters. Although statistically insignificant, the kinetic parameters change in the same fashion as observed for the Tyr125 mutations; $K_{\mathrm{m}, \mathrm{BH} 4}$ decreases, $K_{\mathrm{i}}$ increases, and $k_{\mathrm{cat}, T r p}$ increases. In chTPH2, the loop-swap mutations similarly only introduce modest changes to the kinetic parameters. However, the loopswap mutations in chTPH2 result in introduction of substrate tryptophan inhibition with a $K_{\mathrm{i}}$-value of $497 \pm$ $54 \mu \mathrm{M}$ (Figure 6, panel C black circles). In chTPH1, $K_{\mathrm{i}}$ and $K_{\mathrm{m}, \mathrm{BH} 4}$ seem to be inversely related. This correlates with the observations in loop-swap-chTPH2 where $K_{\mathrm{m}, \mathrm{BH} 4}$ is increased with the introduced substrate inhibition. The conservation of the loops was analyzed (logo plot $^{51}$ in Supporting Information Figure S8) with sequences of TPH1 and TPH2 from 61 and 63 vertebrate species, respectively. It was found that His117, Ala119 and Asn120 of TPH1 are 13, 100, and 97 \% conserved, respectively. In TPH2, Lys163, Ser165, and His166 are conserved 75, 100, and 33 \%, respectively. Of the residues in TPH1, Ala119 and Asn120 show a high degree of conservation, while for TPH2, Ser165 is highly conserved. This implies that these residues are important for proper loop orientation and function. 

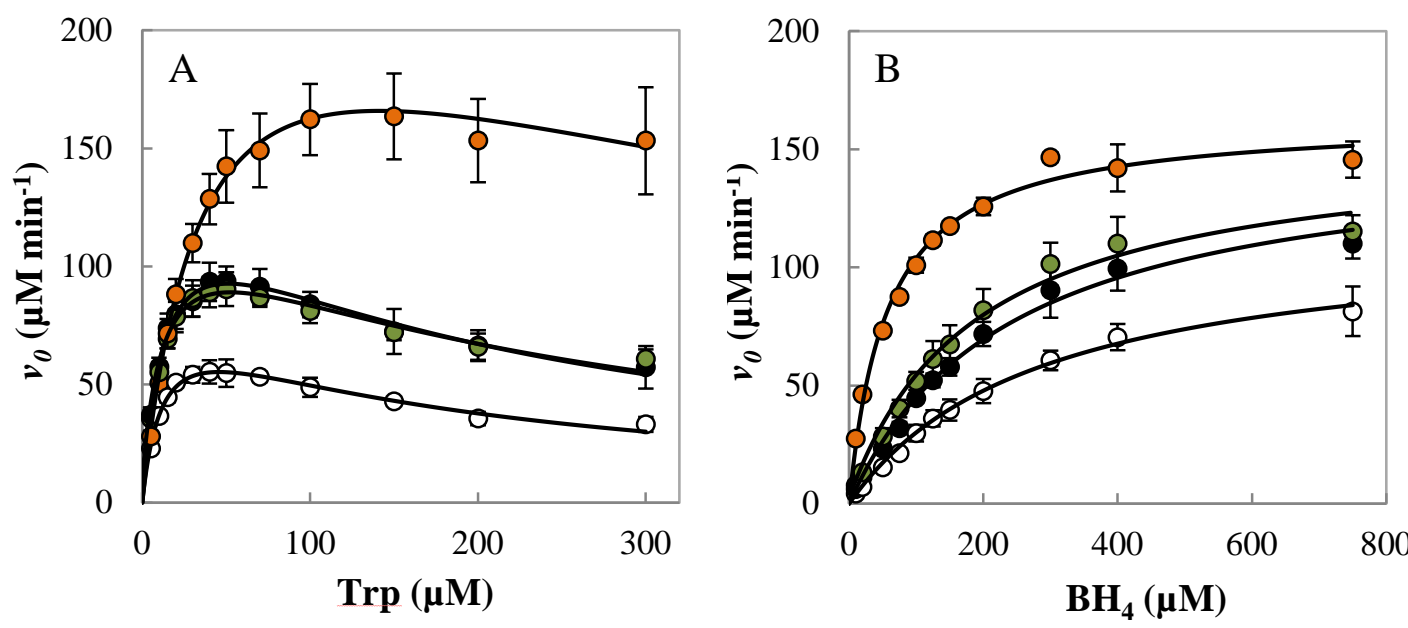

O chTPH1

O Y125A-chTPH1

О Y125W-chTPH1

Loop-swap-chTPH1
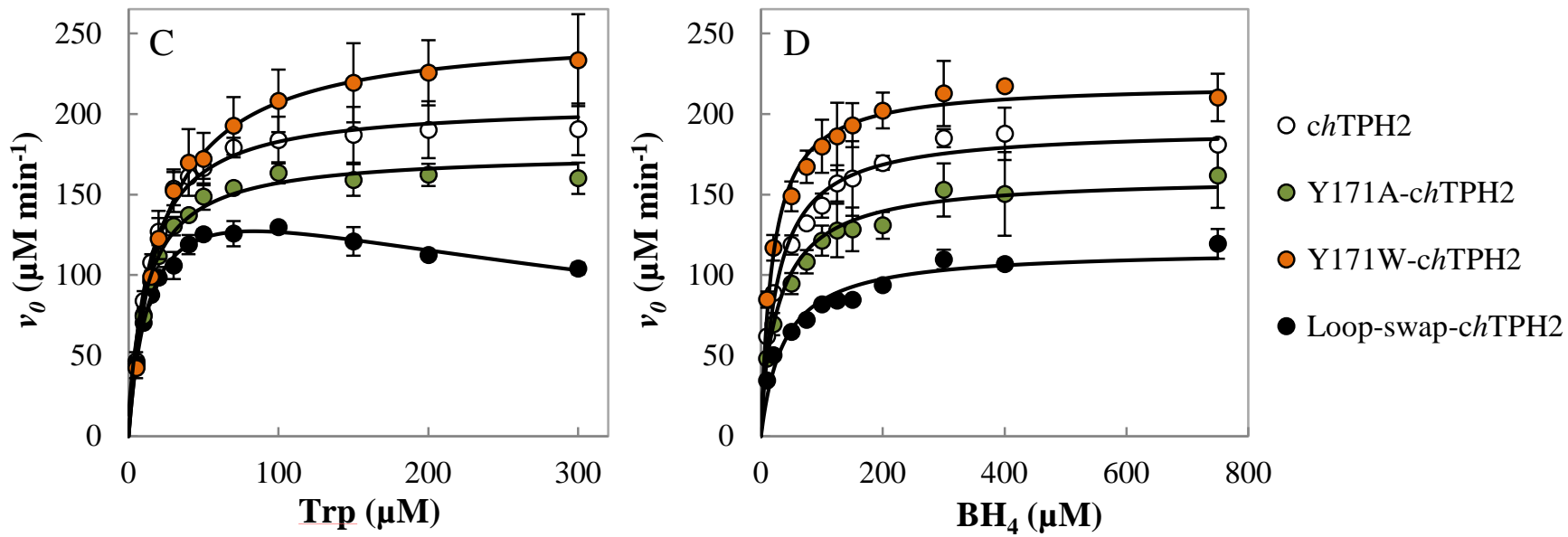

Figure 6. The initial rates of the chTPH1 variants (panel A and B) and of the chTPH2 variants (panel C and D) as a function of Trp or $\mathrm{BH}_{4}$ concentration. The oxygen concentration was $500 \mu \mathrm{M}$ in all measurements. Each measurement is reported as an average with standard deviation of at least three independent experiments from at least two purification batches. The TPH concentration was $0.8 \mu \mathrm{M}$ in all measurements except for Trp at 1.5 and $2.5 \mu \mathrm{M}$, where the concentrations were 0.24 and $0.4 \mu \mathrm{M}$, respectively.

Molecular Dynamics (MD) Simulations - MD simulations were employed to investigate the underlying mechanism that governs the observed substrate inhibition in TPH1. 100 ns MD simulations of chTPH1 with and without substrate Trp were conducted. From comparison of the trajectories, it is observed that in the presence of Trp, the entrance to the $\mathrm{BH}_{4}$ binding pocket becomes blocked, Figure 7 . The blocking of the entrance is caused mainly by Tyr235. Tyr235 is in direct contact with the active site loop through Tyr125, which is under investigation, Figure $8 \mathrm{~A} .{ }^{46}$ Throughout the simulation of chTPH1, Tyr235 resides in a position very similar to the one observed in the crystal structure (average RMSD $\mathrm{Tyr235}_{\text {of }} 1.1 \AA$ compared to 1MLW and 3.6 A compared to 3E2T), Figure 8B. In the simulation of chTPH1 with Trp in the active site, Tyr235 moves to the position which blocks the $\mathrm{BH}_{4}$ entrance. This new position of Tyr235 is very similar to the one observed for the equivalent Tyr236 in the crystal structure of chicken TPH ${ }^{19}$ which has Trp bound (average RMSD $\mathrm{Tyr235}_{\mathrm{T}}$ of $1.8 \AA$ from $3 \mathrm{E} 2 \mathrm{~T}$ and $3.0 \AA$ from $1 \mathrm{MLW}$ ), Figure 8. The movement of Tyr235 observed in the simulation of chTPH1 correlates very well with the structural information obtained from the 
crystal structures. The importance of Tyr235 for the inhibition mechanism of TPH1 is supported by a mutational study, in which it was found that substrate inhibition was removed upon mutation of Tyr235 to alanine or leucine. ${ }^{52}$ In chTPH1, Tyr235 constitutes part of the $\mathrm{BH}_{4}$ binding pocket and is in direct contact with the pterin. ${ }^{46}$ Tyr125 which is under investigation in this study, $\pi$-stacks onto Tyr235 forming a T-shaped interaction. ${ }^{46}$ It is therefore reasonable to believe that mutation of Tyr125 has an influence on the orientation of Tyr235 and thereby explain the influence on the kinetic parameters, especially the substrate inhibition.
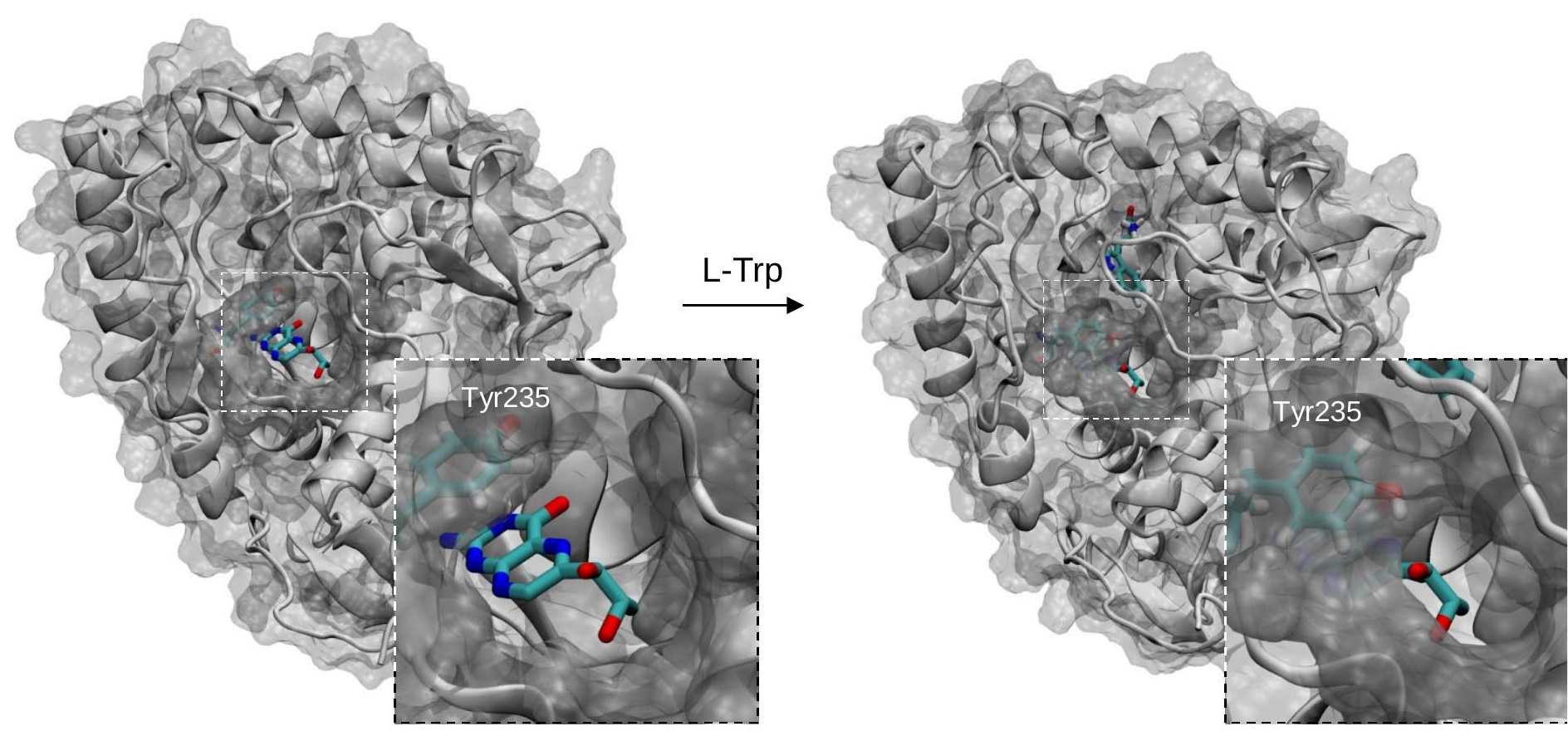

Figure 7. Substrate Trp induced closure of the $\mathrm{BH}_{4}$ binding pocket. $\mathrm{BH}_{2}$, shown in cyan licorice, is not included in the simulations but have been superimposed into the structures (from pdb: 1MLW) to illustrate its binding pose. Left: snapshot extracted from MD simulation of chTPH1 without substrates. Right: snapshot extracted from MD simulation of chTPH1 with bound substrate Trp. The snapshots represent the average position of Tyr235 based on the distance measurements over the 100 ns simulations 
A

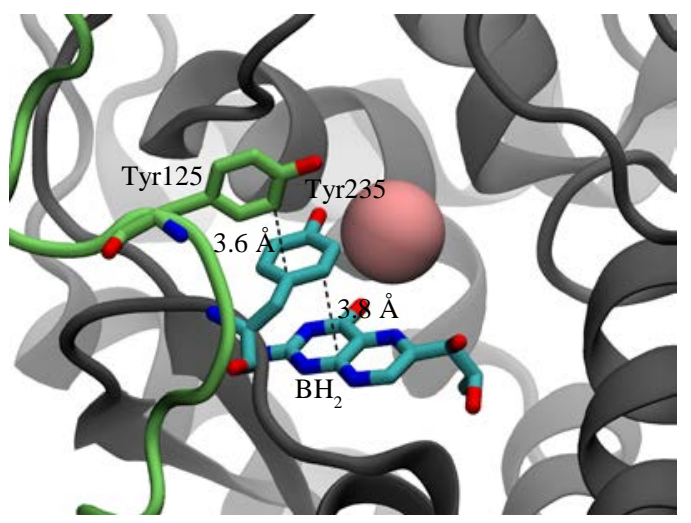

B

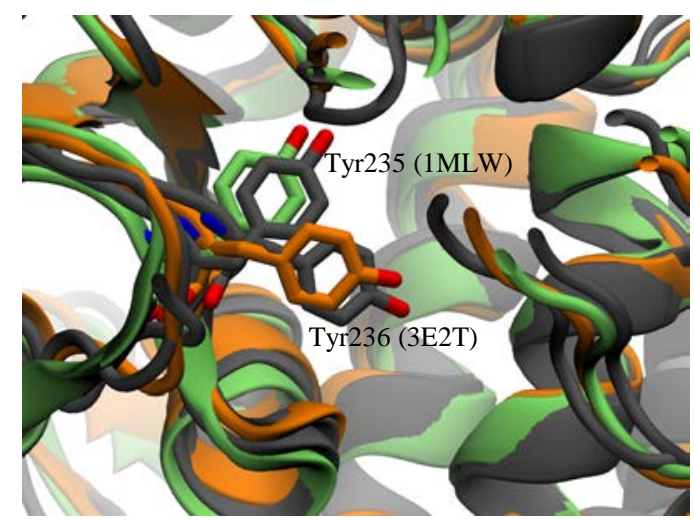

Figure 8. (A) The position and orientation of Tyr125, Tyr235, and $\mathrm{BH}_{2}$ in the crystal structure of chTPH1 (pdb: 1MLW). The active site loop is colored green and dotted lines represent distances. (B) Superposition of the crystal structure of human TPH1 (pdb: 1MLW), chicken TPH1 (pdb: 3E2T), snapshot from MD simulation of chTPH1 without substrate (green Tyr235), and snapshot from MD simulation of chTPH1 with bound Trp (orange Tyr235). The snapshots represent the average position of the tyrosine residues based on the distance measurements over the $100 \mathrm{~ns}$ simulations.

\section{Discussion}

The data presented here shed light on the kinetic mechanism of TPH1 and on the different kinetic properties which are displayed by the TPH isoforms. The kinetic mechanism of TPH has not previously been determined and both Ping Pong and sequential mechanisms have been observed in steady-state kinetic studies of the AAAHs. ${ }^{9-11}$ In this study, a hybrid Ping Pong-ordered mechanism in which the reaction can either occur through a Ping Pong or a sequential mechanism depending on the concentration of tryptophan is observed. The substrate binding order is $\mathrm{BH}_{4}$ followed by tryptophan and substrate inhibition occurs through competitive inhibition of tryptophan versus $\mathrm{BH}_{4}$. This order of substrate binding is in agreement with the majority of studies conducted on the AAAHs. ${ }^{9-12,15-17}$ This model might explain the contradicting results obtained for the AAAHs because very different substrate concentrations are used in these studies.

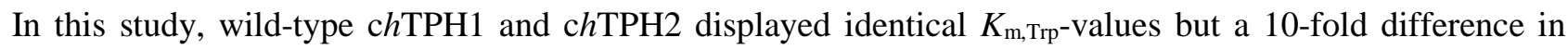
$K_{\mathrm{m}, \mathrm{BH} 4}$-values. Furthermore, a $\sim 2$-fold greater $k_{\text {cat,Trp }}$ was observed for chTPH2 compared to chTPH1 and only chTPH1 displayed substrate inhibition. These kinetic parameters correlate well with previous observation $^{21,25,53}$ although slight variations are seen in some cases. This might be due to that data are based on TPH from different organisms. TPH2 has similarly been reported not to be subject of substrate inhibition $^{1,22,54}$ but has also been found to display inhibition, however, with relatively high inhibition constants $(418-970 \mu \mathrm{M})^{20,55}$. Substrate inhibition is an important regulatory mechanism observed in a variety of enzymes. ${ }^{56}$ Amino acid substrate inhibition has been observed in both full-length human TPH1 and rat TH. ${ }^{57,58}$ Deletion mutagenesis has shown that substrate inhibition is retained to upon deletion of either the regulatory or tetramerization domain of TPH and TH. ${ }^{23,58}$ This suggests that the observed substrate inhibition in full-length TPH and TH only involves the catalytic domain, which implies that the results obtained in this study can be applied to the full-length enzyme. The sequence and structural analyses of the 
two isoforms revealed that the biggest difference between the crystal structures is the tyrosine residue in the active site loop. Mutation of this residue resulted in significant changes in the kinetic parameters of only chTPH1. This implies that this residue has a functional importance in TPH1. The importance of this loop and in particular this residue is supported by findings in PAH and TH where the corresponding residues Tyr138 and Phe184 have been found to be important for proper enzymatic function. ${ }^{59,60}$

Crystal structures of TPH and PAH have clearly revealed the presence of an open and a closed conformation. For PAH, closed conformations have only been observed when both a pterin and a substrate analogue are bound. ${ }^{49,50}$ A closed conformation has also been observed for chicken TPH1 with bound tryptophan and imidazole. ${ }^{19}$ Imidazole is bound in the $\mathrm{BH}_{4}$ binding pocket, which might mimic binding of co-substrate. However, a structure of TPH or PAH with only a substrate/substrate analogue has not been determined, and it is therefore unknown whether the loop closure occurs solely upon binding of substrate or a combination of substrate and co-substrate. The role of the loop closing mechanism is poorly understood. For PAH, it has been suggested that the closing mechanism might protect the co-substrate to enable a high degree of coupling efficiency. ${ }^{59}$ Similarly, the loop in TH has been proposed to exclude water from the active site to avoid reaction of the water with the hydroxylating intermediate before hydroxylation of substrate can occur. ${ }^{48,60,61}$ A similar functional importance of the loop in TPH is supported by the very high sequence conservation among vertebrate species observed for both TPH isoforms. From the MD simulations, it is observed that Trp binding in chTPH1 results in a blocking of the $\mathrm{BH}_{4}$ binding pocket. The Trp-induced blocking mechanism occurs through Tyr235 which moves from a $\mathrm{BH}_{4}$ binding position to a position in the opening of the $\mathrm{BH}_{4}$ binding pocket. This observation compliments the kinetic model in which a dead-end complex is formed when Trp binds before $\mathrm{BH}_{4}$. The influence of Tyr235 on the substrate inhibition mechanism is also supported by the results of Jiang et al. where mutations of Tyr235 to alanine or leucine were found to remove substrate inhibition. ${ }^{52}$ Tyr235 is in direct contact with the active site loop through Tyr125. ${ }^{46}$ The mutations of Tyr125 will therefore most likely have an influence on the orientation of Tyr235, and thereby explain the influence on the kinetic parameters, especially the substrate inhibition. These results strongly suggest that the inhibition mechanism, which is only observed for chTPH1, can be traced to the loop lining the active site. The induced substrate inhibition observed in loop-swap-chTPH2 suggests that the three mutations change the orientation of the loop to an orientation approaching the one observed in chTPH1.

Besides chTPH1, substrate inhibition is also observed in TH. For TH, substrate inhibition is observed at tyrosine concentrations higher than $50 \mu \mathrm{M}^{62}$. The tyrosine concentration in the brain has been found to vary as much as a factor of two. ${ }^{63}$ Substrate inhibition of TH has been proposed to explain the lack of L-DOPA concentration fluctuations as a consequence of increase Tyr concentration upon food intake. ${ }^{56} \operatorname{Trp}$ concentrations are around $60 \mu \mathrm{M}$ in the blood ${ }^{64}$ which is close to the Trp concentration at which the maximum velocity of TPH1 is achieved in this study. This suggests that substrate inhibition could play a role 
in the TPH1 regulation in vivo. It is possible that a corresponding mechanism observed for TH is ensuring that the production of 5-HT does not rise to high levels in the peripheral tissues, which is associated with several diseases. ${ }^{65}$ The concentration of tryptophan in the brain is in the range of $25-35 \mu \mathrm{M}^{66}$ which is close to the observed $K_{\mathrm{m}, \text { Trp }}$ of chTPH2. The velocities of 5-HTP production and in turn serotonin production are, therefore, very sensitive to changes in brain Trp concentration. ${ }^{66,67}$ This might explain the lack of substrate inhibition observed in TPH2, as rapid responses are desirable in the brain. It could be speculated that different regulatory mechanisms of the two isoforms have evolved to accommodate the diverse need for serotonin biosynthesis in different tissues.

\section{Associated content}

\section{Supporting information}

Sequences of $h$ TPH2 protein variants and MBP-3CP. Steady-state kinetic results (Figure S1-S3). Structural analysis of TPH isoform sequence conservation (Figure S4). TPH1 and TPH2 loop densities (Figure S5 and S6). Experimental procedure and results obtained from analytical gel filtration of the TPH variants (Figure S7 and Table S1). Experimental procedure and results obtained from differential scanning fluorimetry measurements conducted on TPH variants (Table S2). Logo conservation plots of the active site loops of the TPH isoforms (Figure S8).

\section{Author Information}

\section{Corresponding authors}

*E-mail: hemc@kemi.dtu.dk

*E-mail: ghp@kemi.dtu.dk

\section{ORCID}

Hans E. M. Christensen: 0000-0002-9540-8679

Günther H. Peters: 0000-0001-9754-2663

\section{Funding}

This work was supported by an Academic Excellence PhD Scholarship to KDT from the Department of Chemistry, Technical University at Denmark.

\section{Notes}

The authors declare no competing financial interest.

\section{Acknowledgements}

The authors would like to thank David F. Nielsen and Martin H. Pedersen for excellent technical assistance. 


\section{Abbreviations}

3CP, 3C-protease; 5-HT, 5-hydroxytryptamin (serotonin); 5-HTP, 5-hydroxytryptophan; AAAH, aromatic amino acid hydroxylase; $\mathrm{BH}_{4}$, tetrahydrobiopterin; DSF, differential scanning fluorimetry; DTT, dithiothreitol; HEPES, 4-(2-hydroxyethyl)piperazine-1-ethanesulfonic acid; MBP, maltose binding protein; MD, molecular dynamics; PAH, phenylalanine hydroxylase; RMSD, root mean square deviation; SEC, size exclusion chromatography; TH, tyrosine hydroxylase; Trp, tryptophan; TPH, tryptophan hydroxylase; chTPH, catalytic domain of human tryptophan hydroxylase.

\section{References}

(1) Carkaci-Salli, N., Flanagan, J. M., Martz, M. K., Salli, U., Walther, D.J., Bader, M., and Vrana, K. E. (2006) Functional domains of human tryptophan hydroxylase 2 (hTPH2), J. Biol. Chem. 281, 28105-28112.

(2) Fitzpatrick, P.F. (1999) Tetrahydropterin-dependent amino acid hydroxylases, Annu. Rev. Biochem. 68, 355-381.

(3) Udenfriend, S., Clark, C. T., and Titus, E. (1953) 5-Hydroxytryptophan decarboxylase: A new route of metabolism of tryptophan, J. Am. Chem. Soc. 75, 501-502.

(4) Liberles, J. S., Thórólfsson, M., and Martínez, A. (2005) Allosteric mechanisms in ACT domain containing enzymes involved in amino acid metabolism, Amino Acids 28, 1-12.

(5) Costagliola, C., Parmeggiani, F., Semeraro, F. and Sebastiani, A. (2008) Selective serotonin reuptake inhibitors: A review of its effects on intraocular pressure, Curr Neuropharmacol. 6, 293-310.

(6) Liu, Q., Yang, Q., Sun, W., Vogel, P., Heydorn, W., Yu, X., Hu, Z., Yu, W., Jonas, B., Pineda, R., Calderon-Gay, V., Germann, M., O’Neill, E., Brommage, R., Cullinan, E., Platt, K., Wilson, A., Powell, D., Sands, A., Zambrowicz, B. and Shi, Z. (2008) Discovery and characterization of novel tryptophan hydroxylase inhibitors that selectively inhibit serotonin synthesis in the gastrointestinal tract, J. Pharmacol. Exp. Ther. 325, 47-55.

(7) Popova, N. K. and Kulikov, A.V. (2010) Targeting tryptophan hydroxylase 2 in affective disorder, Expert Opin. Ther. Targets 14, 1259-1271.

(8) Matthes, S., Mosienko, V., Bashammakh, S., Alenina, N. and Bader, M. (2010) Tryptophan hydroxylase as novel target for the treatment of depressive disorders, Pharmocology 85, 95-109.

(9) Ikeda, M., Fahien, L. A., and Udenfriend, S. (1966) A kinetic study of bovine adrenal tyrosine hydroxylase, J. Biol. Chem. 241, 4452-4456.

(10) Oka, K., Kato, T., Sugimoto, T., Matsuura, S., and Nagatsu, T. (1981) Kinetic properties of tyrosine hydroxylase with natural tetrahydrobiopterin as cofactor, Biochimica et Biophysica Acta 661, 45-53.

(11) Dix, T. A., Kuhn, D. M., Benkovic, S. J. (1987) Mechanism of oxygen activation by tyrosine hydroxylase, Biochemistry 26, 3354-3361.

(12) Fitzpatrick, P. F. (1991) Steady-state kinetic mechanism of rat tyrosine hydroxylase, Biochemistry 30, 3658-3662. 
(13) Chow, M. S., Eser, B. E., Wilson, S. A., Hodgson, K. O., Hedman, B., Fitzpatrick, P. F. and Solomon, E. I. (2009) Spectroscopy and kinetics of wild-type and mutant tyrosine hydroxylase: Mechanistic insight into $\mathrm{O}_{2}$ activation, $J$. Am. Chem. Soc. 131, 7685-7698.

(14) Subedi, B. S. and Fitzpatrick, P. F. (2016) Kinetic mechanism and intrinsic rate constant for the reaction of a bacterial phenylalanine hydroxylase, Biochemistry 55, 6848-6857.

(15) Volner, A., Zoidakis, J., Abu-Omar, M. M. (2003) Order of substrate binding in bacterial phenylalanine hydroxylase and its mechanistic implication for pterin-dependent oxygenases, J. Biol. Inorg. Chem. 8, 121-128.

(16) Roberts, K. M., Pavon, J. A., and Fitzpatrick, P. F. (2013) Kinetic mechanism of phenylalanine hydroxylase: intrinsic binding and rate constants from single-turnover experiments. Biochemistry 52, 1062-1073.

(17) Pavon, J. A., Eser, B., Huynh, M. T., and Fitzpatrick, P. F. (2010) Single turnover kinetics of tryptophan hydroxylase: Evidence for a new intermediate in the reaction of the aromatic amino acid hydroxylases, Biochemistry 49, 7563-7563.

(18) Cianchetta, G., Stouch, T., Yu, W., Shi, Z., Tari, L. W., Swanson, R. V., Hunter, M. J., Hoffman, I. D., and Liu, Q. (2010) Mechanism of inhibition of novel tryptophan hydroxylase inhibitors revealed by co-crystal structures and kinetic analysis, Curr. Chem. Genomics 4, 19-26.

(19) Windahl, M. S., Petersen, C. R., Christensen, H. E. M., and Harris, P. (2008) Crystal structure of tryptophan hydroxylase with bound amino acid substrate, Biochemistry 47(46), 12087-12094.

(20) McKinney, J., Knappskog, P. M., and Haavik, J. (2005) Different properties of the central and peripheral forms of human tryptophan hydroxylase, J. Neurochem. 92, 311-320.

(21) Windahl, M. S., Boesen, J., Karlsen, P. E., and Christensen, H. E. M. (2009) Expression, purification and enzymatic characterization of the catalytic domains of human tryptophan hydroxylase isoforms, Protein J. 28, 400406.

(22) Winge, I., McKinney, J. A., Ying, M., D’Santos, C. S., Kleppe, R., Knappskog, P. M. and Haavik, J. (2008) Activation and stabilization of human tryptophan hydroxylase 2 by phosphorylation and 14-3-3 binding, Biochem. $J$. 410, 195-204.

(23) Yang, X.-J., and Kaufman, S. (1994) High-level expression and deletion mutagenesis of human tryptophan hydroxylase, Proc. Natl. Acad. Sci. 91, 6659-6663.

(24) Walker, P. A., Leong, L. E. C., Ng, P. W. P., Tan, S. H., Waller, S., Murphy, D., and Porter, A. G. (1994) Efficient and rapid affinity purification of proteins using recombinant fusion proteases, Nat. Biotechnol. 12, $601-605$.

(25) Nielsen, M. S., Petersen, C. R., Munch, A., Vendelboe, T. V., Boesen, J., Harris, P., and Christensen, H. E. M. (2008) A simple two step procedure for purification of the catalytic domain of chicken tryptophan hydroxylase 1 in a form suitable for crystallization, Protein Expr. Purif. 57, 116-126.

(26) Tidemand, K. D., Christensen, H. E. M., Hoeck, N., Harris, P., Boesen, J., and Peters, G. H. (2016) Stabilization of tryptophan hydroxylase 2 by L-phenylalanine-induced dimerization, FEBS Open Bio 6, 987-999.

(27) Moran, G. R. and Fitzpatrick, P. F. (1999) A continuous fluorescence assay for tryptophan hydroxylase, Anal. Biochem. 266, 148-152. 
(28) Mannervik, B. (1973) A branching mechanism of glutathione reductase, Biochem Biophys Res Commun. 51, 11511158.

(29) Mannervik, B. (1975) Nonlinear regression methods in design of experiments and mathematical modelling. Applications to the analysis of the steady-state kinetics of glutathione reductase, BioSystems 7, 101-119.

(30) Montero, S., de Arriaga, D., Busto, F., and Soler, J. (1990) A study of the kinetic mechanism followed by glutathione reductase from mycelium of Phycomyces blakesleeanus, Arch. Biochem. Biophys. 278, 52-59.

(31) Schrödinger Release 2016-4: BioLuminate, Schrödinger, LLC, New York, NY, 2016

(32) Schrödinger Suite 2016-4 Protein Preparation Wizard; Epik, Schrödinger, LLC, New York, NY, 2016; Impact, Schrödinger, LLC, New York, NY, 2016; Prime, Schrödinger, LLC, New York, NY, 2016.

(33) Li, H., Robertson, A. D., and Jensen, J. H. (2005) Very fast empirical prediction and rationalization of protein pKa values, Proteins 61, 704-721.

(34) Jorgensen, W. L., Chandrasekhar, J., Madura, J. D., Impey, R. W., and Klein, M. L. (1983) Comparison of simple potential functions for simulating liquid water, J. Chem. Phys. 79, 926-935.

(35) Shivakumar, D., Williams, J., Wu, Y., Damm, W., Shelley, J., and Sherman, W. (2010) Prediction of absolute solvation free energies using molecular dynamics free energy perturbation and the OPLS force field, J. Chem. Theory Comput. 6, 1509-1519.

(36) Harder, E., Damm, W., Maple, J., Wu, C., Reboul, M., Xiang, J. Y., Wang, L., Lupyan, D., Dahlgren, M. K.., Knight, J. L., Kaus, J. W., Cerutti, D., Krilov, G., Jorgensen, W. L., Abel, R., and Friesner, R. A. (2016) OPLS3: a force field providing broad coverage of drug-like small molecules and proteins, J. Chem. Theory Comput., 12, 281-296.

(37) Essmann, U., Perera, L., Berkowitz, M. L., Darden, T., Lee, H., and Pedersen, L. G. (1995) A smooth particle mesh Ewald method, J. Chem. Phys.103, 8577-8593.

(38) Hoover, W. G. (1985) Canonical dynamics: Equilibrium phase-space distributions, Phys. Rev. A 31(3), 1695-1697.

(39) Martyna, G. J., Tobias, D. J., and Klein, M. L. (1994) Constant pressure molecular dynamics algorithms, J. Chem. Phys. 101, 4177-4189.

(40) Humphreys, D. D., Friesner, R. A., and Berne, B. J. (1994) A multiple-time-step molecular dynamics algorithm for macromolecules, J. Phys. Chem. 98 (27), 6885-6892.

(41) Leskovac, V. (2003) Comprehensive enzyme kinetics, Kluwer Academic/Plenum Press, New York.

(42) Segel, I. H., (1975) Enzyme kinetics - Behavior and analysis of rapid equilibrium and steady-state enzyme systems. John Wiley and Son, Inc., New York.

(43) Krenitsky, T. A., Papaioannou, R. (1969) Human hypoxanthine phosphoribosyltransferase, J. Biol. Chem. 244, 1271-1277.

(44) McClure, W. R., Lardy, H. A., and Kneifel, H. P. (1971) Rat liver pyruvate carboxylase, J. Biol. Chem. 246, 35693578.

(45) Pabst, M. J., Habig, W. H., and Jakoby, W. B. (1974) Glutathione S-transferase, J. Biol. Chem. 249(22), 71407150. 
(46) Wang, L., Erlandsen, H., Haavik, J., Knappskog, P. M. and Stevens, R.C. (2002) Three-dimensional structure of human tryptophan hydroxylase and its implications for the biosynthesis of the neurotransmitters serotonin and melatonin, Biochemistry 41, 12569-12574.

(47) Erlandsen, H., Bjørgo, E., Flatmark, T., Stevens, R. C. (2000) Crystal structure and site-specific mutagenesis of pterin-bound human phenylalanine hydroxylase, Biochemistry 39, 2208-2217.

(48) Andersen, O. A., Flatmark, T., and Hough, E. (2001) High resolution crystal structures of the catalytic domain of human phenylalanine hydroxylase in its catalytically active Fe(II) form and binary complex with tetrahydrobiopterin, $J$. Mol. Biol. 314, 279-291.

(49) Andersen, O. A., Flatmark, T., and Hough, E. (2002) Crystal structure of the ternary complex of the catalytic domain of human phenylalanine hydroxylase with tetrahydrobiopterin and 3-(2-thienyl)-L-alanine, and its implications for the mechanism of catalysis and substrate activation, J. Mol. Biol. 320, 1095-1108.

(50) Andersen, O. A., Stokka, A. J., Flatmark, T., and Hough, E. (2003) 2.0 Å resolution crystal structures of the ternary complexes of human phenylalanine hydroxylase catalytic domain with tetrahydrobiopterin and 3-(2-thienyl)-L-alanine or L-norleucine: substrate specificity and molecular motions related to substrate binding, J. Mol. Biol. 333, 747-757.

(51) Crooks, G.E., Hon, G., Chandonia, J. M., Brenner, S.E. (2004) WebLogo: A sequence logo generator, Genome Research 14, 1188-1190.

(52) Jiang, G. C. T., Yohrling, G. J., Schmitt, I. V. J. D., and Vrana, K. E. (2000) Identification of substrate orienting and phosphorylation sites within tryptophan hydroxylase using homology-based molecular modeling, J. Mol. Biol. 302, 1005-1017.

(53) Moran, G. R., Daubner, S.C., and Fitzpatrick, P. F. (1998) Expression and characterization of the catalytic core of tryptophan hydroxylase, J. Biol. Chem. 273, 12259-12266.

(54) Cichon, S., Winge, I., Mattheisen, M., Georgi, A., Karpushova, A., Freudenberg, J., Freudenberg-Hua, Y., Babadjanova, G., Van Den Bogaert, A., Abramova, L. I., Kapiletti, S., Knappskog, P. M., McKinney, J., Maier, W., Jamra, R. A., Schulze, T. G., Schumacher, J., Propping, P., Rietschel, M., Haavik, J., and Nöthen, M. M. (2008) Brainspecific tryptophan hydroxylase 2 (TPH2): a functional Pro206Ser substitution and variation in the 5'-region are associated with bipolar affective disorder, Hum. Mol. Genet. 17, 87-97.

(55) Winge, I., McKinney, J. A., Knappskog, P. M., and Haavik, J. (2007) Characterization of wild-type and mutant forms of human tryptophan hydroxylase 2, J. Neurochem. 100, 1648-1657.

(56) Reed, M. C., Lieb, A., and Nijhout, H. F. (2010) The biological significance of substrate inhibition: A mechanism with diverse functions, Bioessays 32, 422-429.

(57) McKinney, J., Knappskog, P. M., Pereira, J., Ekern, T., Toska, K., Kuitert, B. B., Levine, D., Gronenborn, A. M., Martinez, A., and Haavik, J. (2004) Expression and purification of human tryptophan hydroxylase from Escherichia coli and Pichia pastoris, Protein Expr. Purif. 33, 185-194. 
(58) Ribeiro, P., Wang, Y., Citron, B. A., and Kaufman, S. (1993) Deletion mutagenesis of rat PC12 tyrosine hydroxylase regulatory and catalytic domains. J. Molec. Neurosci. 4, 125-139.

(59) Leandro, J., Stokka, A. J., Teigen, K., Andersen, O. A., Flatmark, T. (2017) Substituting Tyr138 in the active site loop of human phenylalanine hydroxylase affects catalysis and substrate activation, FEBS Open Bio 7, 1026-1036.

(60) Daubner, S. C., McGinnis, J. T., Gardner, M., Kroboth, S. L., Morris, A. R., and Fitzpatrick, P. F. (2006) A flexible loop in tyrosine hydroxylase controls coupling of amino acid hydroxylation to tetrahydropterin oxidation, J. Mol. Biol. 359, 299-307.

(61) Frantom, P. A. and Fitzpatrick, P. F. (2003) Uncoupled forms of tyrosine hydroxylase unmask kinetic isotope effects on chemical steps, J. Am. Chem. Soc. 125, 16190-16191.

(62) Quinsey, N. S., Luong, A. Q., and Dickson, P. W. (1998) Mutational analysis of substrate inhibition in tyrosine hydroxylase, J. Neurochem. 71, 2132-2138.

(63) Fernstrom, J. D. and Faller, D. V. (1978) Neutral amino acids in the brain: changes in response to food ingestion, J. Neurochem. 30, 1531-1538.

(64) Breum, L., Rasmussen, M. H., Hilsted, J., and Fernstrom, J. D. (2003) Twenty-four-hour plasma tryptophan concentrations and ratios are below normal in obese subjects and are not normalized by substantial weight reduction, Am. J. Clin. Nutr. 77, 1112-1118.

(65) Amireault, P., Sibon, D., and Coté, F. (2013) Life without peripheral serotonin: Insights from tryptophan hydroxylase 1 knockout mice reveal the existence of paracrine/autocrine serotonergic networks, ACS Chem. Neurosci. 4, 64-71.

(66) Fernstrom, J. and Fernstrom, M. (1995) Brain tryptophan concentrations and serotonin synthesis remain responsive to food consumption after the ingestion of sequential meals, Am. J. Clin. Nutr. 61, 312-319.

(67) Knott, P. J. and Curzon, G. (1972) Free tryptophan in plasma and brain tryptophan metabolism, Nature 239, 452453. 